\title{
How Do Physiotherapists Treat People with Knee Osteoarthritis and their evidence awareness: A cross-sectional survey among Indian Physiotherapists
}

Pranav Saha ${ }^{1}$, Tenzin Yangchen ${ }^{1}$, Shaili Sharma ${ }^{1}$, Jaspinder Kaur ${ }^{1}$, Tsetan Norboo ${ }^{1}$, Ammar Suhail *2.

${ }^{1}$ BPT $4^{\text {th }}$ Year, Department of Physiotherapy, Lovely Professional University, Phagwara, Punjab, India.

${ }^{* 2}$ Assistant Professor, Department of Physiotherapy, Lovely Professional University, Phagwara, Punjab, India.

\section{ABSTRACT}

Background: Evidence-based practice in the field of physiotherapy is of utmost importance and is the way ahead. Physiotherapists' knowledge of evidence in terms of assessment and management of knee OA may influence the implementation of evidence-based practice. However, physiotherapist's practice patterns are usually based on their professional experience or expert opinions. The present study aimed to explore the evidence awareness among Indian physiotherapists and identify their practice patterns in patients with knee OA.

Methods: An online cross-sectional survey was conducted among Indian physiotherapists. The survey was designed using current clinical practice guidelines and previously published similar studies. The survey consisted of three sections: (1) demographic details, (2) questions related to evidence awareness, (3) questions related to the practice patterns of Indian physiotherapists. The evidence awareness and practice patterns of physiotherapists were explored.

Results: A total of 165 responses were received and analyzed. Most participants perceived footwear modifications $(21.2 \%)$ and resistance exercises $(44.2 \%)$ as having very strong evidence. Range of motion assessment and functional assessment was regarded as a must during the patient's initial visit. In terms of management strategies majority of the participants considered patient education $(n=122)$, strengthening exercises $(n=81)$, and therapeutic ultrasound $(n=79)$ as their treatment of choice.

Conclusion: Most physiotherapist assessment strategies were aimed at identifying impairments in patients. In line with the clinical practice guidelines, most physiotherapists use patient education and strengthening exercises to treat patients with knee OA. However, treatment modalities like ultrasound, TENS, and dry needling, which lack scientific support, are still being used by the physiotherapist. There is a lack of coherence between physiotherapists' evidence awareness and their choice of management strategies.

KEY WORDS: Knee, Osteoarthritis, Survey, Resistance exercises.

Address for correspondence: Ammar Suhail, Assistant Professor, Department of Physiotherapy Lovely Professional University, Phagwara, Punjab, India. Phone no: +91-9718254388

E-Mail: asuhail38@gmail.com,ammar.19815@lpu.co.in

Access this Article online

Quick Response code

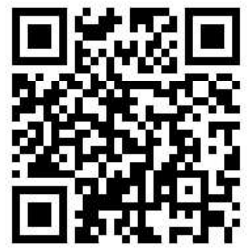

DOI: $10.16965 / \mathrm{ijpr} .2021 .161$

Journal Information

International Journal of Physiotherapy and Research ISSN (E) 2321-1822 | ISSN (P) 2321-8975

https://www.ijmhr.org/ijpr.html

DOI-Prefix: https://dx.doi.org/10.16965/ijpr

\section{Article Information}

Received: 25 Jun 2021

Peer Review: 26 Jun 2021

Revised: None
Accepted: 03 Aug 2021

Published (O): 11 Aug 2021

Published (P): 11 Aug 2021
INTRODUCTION

Osteoarthritis (OA) is a prevalent disorder of synovial joints characterized by structural changes in hyaline articular cartilage, subchondral bone, ligaments, capsule, synovium, and periarticular muscles [1]. Knee is the most 
commonly involved joint, affecting $37 \%$ of the population of 60 years and older, resulting in significant personal and societal impact [2-4]. In the Framingham study, the prevalence of radiographic knee OA was $19.2 \%$ and $27.8 \%$ in the Johnston County Osteoarthritis Project $[5,6]$. Cui et al. reported the global prevalence of knee OA as $22.9 \%$ among individuals in $\geq 40$ years [7].

Although knee $O A$ is a growing public health problem, there is a lack of evidence on the prevalence of knee OA among the Indian population. However, Pal et al. report a prevalence of $28.7 \%$ in their study conducted among five sites in India [8]. Symptomatic knee $O A$ is defined as the presence of pain, aching, or stiffness in a joint with radiographic OA [9]. Patients usually present with pain, stiffness, reduced joint motion, and muscle weakness [2]. Pain is the primary complaint and is often the reason to seek medical care $[10,11]$.

Management of impairments associated with knee $O A$ requires a multidisciplinary approach that involves pharmacological and nonpharmacological treatment $[12,13]$.

Physiotherapy interventions are a mainstay of the non-pharmacological management of knee OA [10]. In addition, recent clinical practice guidelines published by major International bodies emphasize evidence-based management strategies [14-18].

Evidence-based practice in the field of physiotherapy is of utmost importance and well established. However, there are fewer studies that explore whether physiotherapists use evidence-backed management or not. For example, Spitaels and colleagues reported a lack of adherence to evidence-based quality indicators [19]. Another study conducted in Canada had similar findings; they found that modalities lacking evidence are still being used by physiotherapists [20].

The physiotherapists' practice varies across countries and depends on the healthcare setup. There is a dearth of studies from developing countries like India exploring practice patterns of physiotherapists. Therefore, the purpose of this study was to explore the vperspectives of physiotherapists regarding the strength of evidence of effectiveness for commonly used strategies in the management of knee OA. The study also investigates the assessment and management choices of a physiotherapist.

\section{METHODS}

The study was conducted exclusively online and used a cross-sectional research design. The online survey used google forms to collect the data through social media handles. Physiotherapists with at least an undergraduate professional degree and more than one year of practice were considered as eligible respondents. Physiotherapy students and interns were excluded from the survey.

The survey was divided into three sections. The first section contained basic information about the study and a consent form with other demographic questions too. The second section had questions that asked them to rate how strongly they felt the research evidence is available for a list of currently used treatments for knee OA. The survey instrument questions were designed to explore the evidence knowledge of the respondents. The management strategies as questions could be rated as 'very strong evidence,' 'strong evidence,' 'moderate evidence,' weak evidence, and 'very weak evidence.'

The third section consisted of a clinical scenario, and respondents were asked their preferred assessment and management strategies for the given scenario. The responses were open-ended to ensure the exploration of the commonly employed techniques by the physiotherapists.

The project approval committee of the Department of Physiotherapy at Lovely Professional University approved the study's protocol. It adhered to the ethical principles for medical research involving human subjects described by the WMA Declaration of Helsinki. This was an exploratory pilot survey conducted among practicing Indian physiotherapists. The study is part of a project aimed at exploring different domains of Knee OA [21].

The data was collected from March to May 2021. Later, the collected information was converted into a master excel sheet for analysis 
using Microsoft excel and reported using descriptive statistics.

\section{RESULTS}

A total of 175 physiotherapists participated in the study. Ten participants only filled in demographic details and did not provide consent; therefore, 165 participants' data was finally analyzed. The mean age of the participants was 26.9 years \pm 6.09 . All the participants testified that they regularly treat Knee OA patients. The demographic characteristics are highlighted in Table 1.

Table 1: Demographic characteristics of the participants.

\begin{tabular}{lcc}
\hline \multicolumn{1}{c}{ Variables } & Mean & Standard Deviation \\
\hline Mean (SD) age (years) & 26.9 & 6.09 \\
& Frequency (n) & Percentage (\%) \\
Male & 61 & 37 \\
Female & 108 & 63 \\
Education Level & & \\
BPT & 96 & 58.2 \\
MPT & 65 & 39.4 \\
PhD & 4 & 2.4 \\
Years of Practice & & \\
1 to 5 years & 132 & 80 \\
6 to 10 years & 20 & 12.8 \\
11 to 15 years & 9 & 5.5 \\
16 to 20 years & 4 & 2.4 \\
Work Setup & & \\
Private Clinic & 68 & 41.2 \\
Teaching/Research & 24 & 14.6 \\
Institute & & 33.3 \\
Private Hospital & 55 & 10.3 \\
Government Hospital & 17 & 0.6 \\
Government Clinic & 1 & \\
\hline
\end{tabular}

In section one, reported evidence levels of effectiveness shows high variability in responses for commonly used management strategies.

Ergonomic advice as a management strategy achieved $44.2 \%(n=73)$ of participants stating it in the "very strong evidence" category. Participants considered resistance exercises to be the second-best evidence-based management strategy as $21.2 \%(n=35)$ agreed to it. Manual therapy directed at joint put up in strong evidence section by $43.8 \%(n=71)$.

Electrophysical agents were also rated to have strong evidence in management in knee OA patients. Knee bracing was reported as strong evidence by $34.5 \%(n=57)$, while $33.9 \%(n-=56)$ said it to have moderate evidence. Table 1 and figure 1 illustrates the participants reported evidence levels for the different management strategy.

In the second section, the clinical scenario was followed by the question, "During the first visit with this patient, what assessment strategy would you use?" Most participants ( $n=129$ ), $78.2 \%$, reported using range of motion assessment (ROM). Participants suggested different assessment strategies; figure 2 shows the various assessment strategies.

For the second question, "During the first visit with this patient, what therapy would you recommend/provide as a Core treatment."

Table 2: Treatments for knee OA with reported evidence levels of effectiveness. Figures are presented as a percentage (\%) of the valid responses $(n=165)$.

\begin{tabular}{lccccc}
\hline \multicolumn{1}{c}{ Treatments } & $\begin{array}{c}\text { Very Strong } \\
\text { evidence }\end{array}$ & $\begin{array}{c}\text { Strong } \\
\text { evidence }\end{array}$ & $\begin{array}{c}\text { Moderate } \\
\text { evidence }\end{array}$ & $\begin{array}{c}\text { Weak } \\
\text { evidence }\end{array}$ & $\begin{array}{c}\text { Very weak } \\
\text { evidence }\end{array}$ \\
\hline General exercises & 20 & 44.2 & 29.7 & 4.9 & 1.2 \\
Aerobic Exercises & 10.9 & 30.3 & 43.6 & 8.5 & 6.7 \\
Resistance Exercises & 21.2 & 48.5 & 25.5 & 4.2 & 0.6 \\
TENS/IFT & 12.1 & 35.2 & 34.5 & 15.2 & 3 \\
Ultrasound & 15.1 & 28.5 & 36.4 & 16.4 & 3.6 \\
Knee Bracing & 11.5 & 34.5 & 34 & 15.8 & 4.2 \\
Body weight supported treadmill training & 7.9 & 24.2 & 44.2 & 14.6 & 9.1 \\
(BWSTT) & 9.1 & 44.2 & 24.2 & 14.6 & 7.9 \\
\hline Weight loss & 44.2 & 32.1 & 17 & 6.1 & 0.6 \\
\hline Footwear Modification & 17.3 & 43.8 & 24.7 & 11.1 & 3.1 \\
\hline Manual therapy directed at the joint & 12.3 & 38.9 & 36.4 & 6.8 & 5.6 \\
\hline Manual therapy directed at soft tissues & & & & & \\
\hline
\end{tabular}

Patient education was the most widely preferred therapy opted by the participants chosen by $(n=115) 69.7 \%$ of participants. $\quad(n=81)$. Figure 3 shows the different treatment Strengthening exercises were the second most strategies suggested by the participants. 


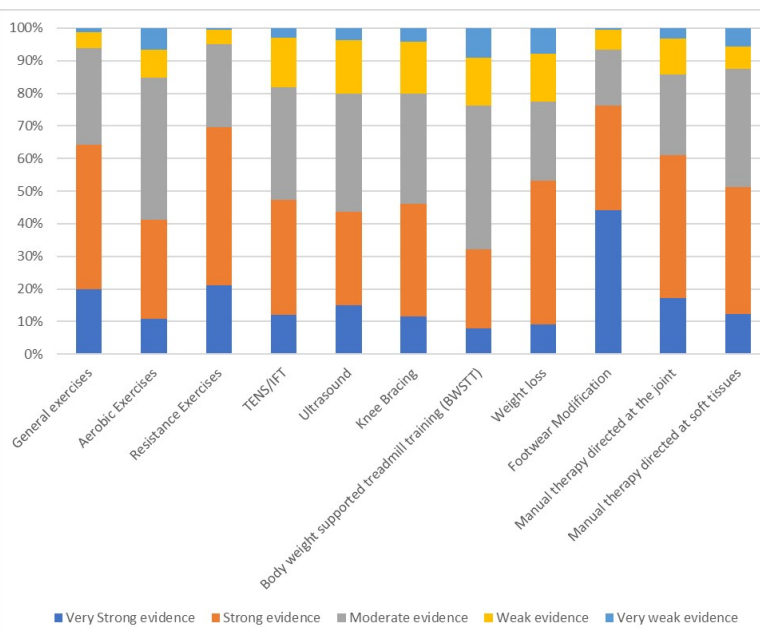

Fig. 1: Effectiveness of different management strategies rated by the participants Section Two: Participant's choice of assessment and management strategy.

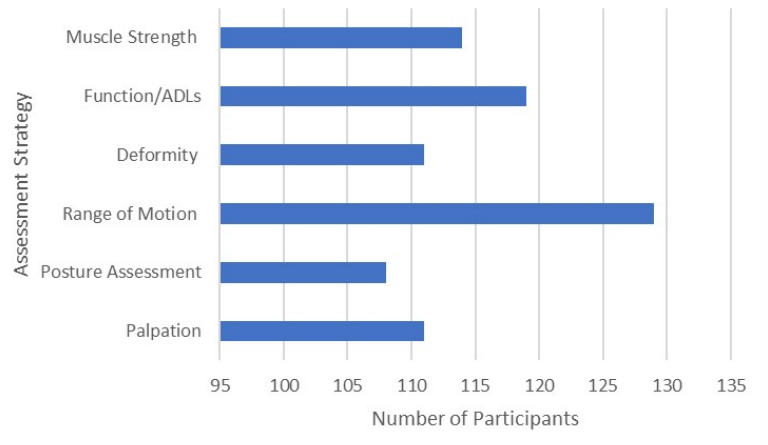

Fig. 2: Assessment approaches used by physiotherapists for knee OA.

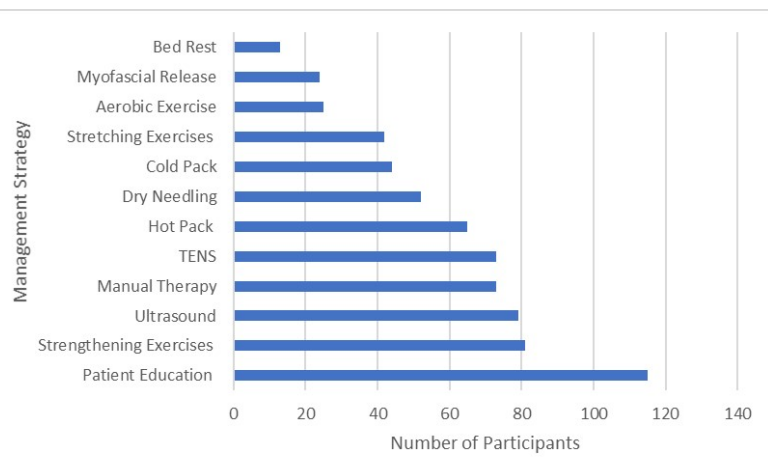

Fig. 3: Treatment approaches used by physiotherapists for knee OA.

\section{DISCUSSION}

The study's main aim was to assess the physiotherapists' perspective about the level of evidence of the effectiveness of different management strategies. The data analysis revealed varied responses with footwear modification as the only strategy rated as "very strong evidence" by $44.2 \%$ of participants, followed by "resistance exercises" ranked second at $21.2 \%$. The study's secondary objectives were to explore the choices of assessment and management modalities commonly used by physiotherapists. These strategies were assessed using open-ended questions, which yielded patient education as an essentiality in the initial visit by majority of participants. While choosing assessment strategies for knee OA patients, most participants opted for impairment identification methods such as an ROM assessment $(n=129)$. Other techniques identified were the evaluation of muscle strength, functional capacity, and posture.

The participants rated exercises as having strong evidence in the management of knee $\mathrm{OA}$. There was a negligible difference in the perceived strength of evidence among the different forms of exercise. However, resistance exercises $(n=81)$ were preferred over other forms of exercises. This predilection was as major clinical practice guidelines (CPG), which recommend exercise as the first line of treatment in knee OA [14-16]. However, the evidence for the "best" exercise for knee OA patients is inconclusive and is usually driven by expert opinions $[14,18]$. Participants suggested stretching exercises as a management tool, which may be clinically beneficial but lacks evidence support.

The research on the type of exercise is highly heterogeneous, with many variations in the dosimetry of exercise. Evidently, both aerobic and resistance exercises are beneficial in pain reduction and function. The Ottawa panel CPG suggests that resistance exercises must be combined with behavioural strategies to enhance adherence and improve long-term outcomes [17]. Despite rating exercise in the strong evidence category, fewer participants recommend it as a core strategy. This may be due to a lack of experience and confidence in prescribing a structured exercise protocol. A physiotherapist must be well versed with exercise prescription and must tailor an exercise program to individual patients. Educational programs must incorporate exercise prescription as a module too.

Electrophysical agents like ultrasound and TENS/IFT were considered to have strong to moderate evidence. Ultrasound was the most popular modality $(n=79)$, followed by TENS 
Pranav Saha, Tenzin Yangchen, Shaili Sharma, Jaspinder Kaur, Tsetan Norboo, Ammar Suhail. How Do Physiotherapists Treat People with Knee Osteoarthritis and their evidence awareness: A cross-sectional survey among Indian Physiotherapists.

$(n=71)$. American College of Rheumatology strongly recommends against the usage of TENS/IFT [14]. Despite the negative evidence, TENS/IFT, Ultrasound, and Hot/cold packs were preferred by participants to manage knee $\mathrm{OA}$. These modalities are believed to produce very short-lived effects, and studies have failed to demonstrate their efficacy.

In terms of unloading strategies, i.e., weight loss, knee brace, and body weight treadmill training were reported to have strong to moderate evidence. Weight loss as a treatment strategy is recommended across different CPGs [14,15,22]. A dose-response relationship is reported for weight loss; a loss of $10-19.9 \%$ of baseline body weight is associated with changes in clinical and mechanistic outcomes [23]. The weight loss is also related to other health benefits and must be incorporated in management strategies for knee OA. Knee bracing is also strongly recommended, which resonated with participants' responses as the majority graded it as strong to moderate evidence. Knee braces in the early stages can significantly impact the ambulation of patients [14].

Bodyweight support treadmill training (BSWTT) is not in recommendations in any of the CPGs, as research is still lacking for BSWTT in knee $\mathrm{OA}$; moreover, the cost of the equipment makes it useable only in select setting most of the participants considered BSWTT to have moderate evidence. While choosing treatment strategies, participants did not mention any unloading management modalities. Footwear was believed to have strong evidence by the participants. Although the evidence is scarce, a good and comfortable shoe should be recommended for patients with knee OA [15]. Unfortunately, the survey did not specify internal shoe modifications, so the data could not indicate whether participants referred to shoe modifications or advice for a comfortable shoe.

Manual therapy, either targeting joints or soft tissues, is recommended "conditionally against" in the ACR guidelines, which differed significantly from the survey participants' opinions [14]. The survey participants agreed that manual therapy has strong to moderate evidence. Literature suggests that manual therapy must be used as an adjunct along with other interventions and should not be used as a stand-alone treatment.

In addition, participants also reported using bed rest and patient education as a treatment option. Although only 13 participants mentioned bed rest, it still is an alarming finding. Bed rest should not be promoted among patients with knee $O A$ and therapists must be educated about the same. The most positive finding of the study was that physiotherapist suggests patient education as one of the essential treatment options ( $n=115)$. However, this was confounded with $79.4 \%$ of the participants believed that knee $O A$ is a "wear and tear" problem. Future studies must evaluate the beliefs of physiotherapists about knee $O A$ and its impact on their clinical practice.

The findings of our study are similar to previous studies conducted on the same theme. For example, in a study by Spitaels and colleagues, partaken physiotherapists provided education on the importance of exercise, delivering functional and strength exercise therapy and patient-tailored exercise program. They also reported using treatment modalities with low-value care like massage and ice [19].

Another similar study conducted in Canada reported that physiotherapists use therapeutic exercise and education to treat people with knee OA; however, electrophysical agents like ultrasound are still in use. These findings are quite like the current study, and participants preferred exercise and patient education. On the other hand, low evidence modalities were still in practice and frequently suggested.

The study has various strengths and limitations. The key strength of the study is that it identified different ways of practicing Indian physiotherapists. Although the sample size is less, the findings are particularly important as they may guide us in improving the training of practicing physiotherapists. The major limitation was the short duration of data collection which resulted in a smaller number of participants. 
Pranav Saha, Tenzin Yangchen, Shaili Sharma, Jaspinder Kaur, Tsetan Norboo, Ammar Suhail. How Do Physiotherapists Treat People with Knee Osteoarthritis and their evidence awareness: A cross-sectional survey among Indian Physiotherapists.

\section{CONCLUSION}

Study participants mainly used patient education and exercises to treat patients with knee OA. In terms of assessment range of motion, functional status and posture was opted by the study participants. The management choices were in line with the recent practice guidelines, however modalities that lack evidence are still used by the study participants. Education programs should focus on disseminating the evidence among the practicing physiotherapists.

\section{ACKNOWLEDGEMENTS}

We would like to thank all the physiotherapists who participated in the survey.

Source of Funding: This research did not receive any specific grant from funding agencies in the public, commercial, or not for-profit sectors.

\section{Conflicts of interest: None}

\section{REFERENCES}

[1]. Hunter DJ, Bierma-Zeinstra S. Osteoarthritis. Lancet 2019; 393: 1745-59.

[2]. Sharma L. Osteoarthritis of the Knee. N Engl J Med 2021; 384: 51-9.

[3]. Mahendira L, Jones C, Papachristos A, Waddell J, Rubin L. Comparative clinical and cost analysis between surgical and non-surgical intervention for knee osteoarthritis. Int Orthop 2020; 44: 77-83.

[4]. Martel-Pelletier J, Barr AJ, Cicuttini FM, et al. Osteoarthritis. Nat Rev Dis Prim 2016; 2. DOI:10.1038/ nrdp.2016.72.

[5]. Jordan JM, Helmick CG, Renner JB, et al. Prevalence of Hip Symptoms and Radiographic and Symptomatic Hip Osteoarthritis in African Americans and Caucasians: The Johnston County Osteoarthritis Project. J Rheumatol 2009; 36: 809-15.

[6]. Felson DT, Naimark A, Anderson J, Kazis L, Castelli W, Meenan RF. The prevalence of knee osteoarthritis in the elderly. the framingham osteoarthritis study. Arthritis Rheum 1987; 30: 914-8.

[7]. Cui A, Li H, Wang D, Zhong J, Chen Y, Lu H. Global, regional prevalence, incidence and risk factors of knee osteoarthritis in population-based studies. EClinicalMedicine 2020; 29-30: 100587.

[8]. Pal CP, Singh P, Chaturvedi S, Pruthi KK, Vij A. Epidemiology of knee osteoarthritis in India and related factors. Indian J Orthop 2016; 50: 518-22.

[9]. Zhang Y, Jordan JM. Epidemiology of Osteoarthritis. Rheum Dis Clin North Am 2008; 34: 515-29.
[10]. Ayanniyi O, Egwu RF, Adeniyi AF. Physiotherapy management of knee osteoarthritis in Nigeria-A survey of self-reported treatment preferences. Hong Kong Physiother J 2017; 36: 1-9.

[11]. Neogi T. The epidemiology and impact of pain in osteoarthritis. Osteoarthr Cartil 2013; 21: 1145-53.

[12]. James SL, Abate D, Abate KH, et al. Global, regional, and national incidence, prevalence, and years lived with disability for 354 Diseases and Injuries for 195 countries and territories, 1990-2017: A systematic analysis for the Global Burden of Disease Study 2017. Lancet 2018; 392: 1789-858.

[13]. Zhang W, Doherty M, Peat G, et al. EULAR evidencebased recommendations for the diagnosis of knee osteoarthritis. Ann Rheum Dis 2010; 69: 483-9.

[14]. Kolasinski SL, Neogi T, Hochberg MC, et al. American College of Rheumatology/Arthritis Foundation Guideline for the Management of Osteoarthritis of the Hand, Hip, and Knee. Arthritis Rheumatol 2020; 72: 220-33.

[15]. Fernandes L, Hagen KB, Bijlsma JWJ, et al. EULAR recommendations for the non-pharmacological core management of hip and knee osteoarthritis. Ann Rheum Dis 2013; 72: 1125-35.

[16]. Bannuru RR, Osani MC, Vaysbrot EE, et al. OARSI guidelines for the non-surgical management of knee, hip, and polyarticular osteoarthritis. Osteoarthr Cartil 2019; 27: 1578-89.

[17]. Brosseau L, Taki J, Desjardins B, et al. The Ottawa panel clinical practice guidelines for the management of knee osteoarthritis. Part two: Strengthening exercise programs. Clin Rehabil 2017; 31: 596-611.

[18]. Brosseau L, Taki J, Desjardins B, et al. The Ottawa panel clinical practice guidelines for the management of knee osteoarthritis. Part three: Aerobic exercise programs. Clin Rehabil 2017; 31: 612-24.

[19]. Spitaels D, Hermens R, Van Assche D, Verschueren S, Luyten F, Vankrunkelsven P. Are physiotherapists adhering to quality indicators for the management of knee osteoarthritis? An observational study. Musculoskelet Sci Pract 2017; 27: 112-23.

[20]. da Costa BR, Vieira ER, Gadotti IC, et al. How do physical therapists treat people with knee osteoarthritis, and what drives their clinical decisions? A population-based cross-sectional survey. Physiother Canada 2017; 69: 30-7.

[21].Suhail A. Knee Osteoarthritis. 2021. https:// www.researchgate.net/project/Knee-Osteoarthritis-8 (accessed June 15, 2021).

[22]. Manara M, Bortoluzzi A, Favero $M$, et al. The Italian Society for Rheumatology clinical practice guidelines for the diagnosis and management of knee, hip and hand osteoarthritis. Reumatismo 2019; 65: 4-21.

[23]. Messier SP, Resnik AE, Beavers DP, et al. Intentional Weight Loss in Overweight and Obese Patients With Knee Osteoarthritis: Is More Better? Arthritis Care Res (Hoboken) 2018; 70: 1569-75.

How to cite this article: Pranav Saha, Tenzin Yangchen, Shaili Sharma, Jaspinder Kaur, Tsetan Norboo, Ammar Suhail. How Do Physiotherapists Treat People with Knee Osteoarthritis and their evidence awareness: A crosssectional survey among Indian Physiotherapists. Int J Physiother Res 2021;9(4):3968-3973. DOI: 10.16965/ ijpr.2021.161 